CARDIOVASCULAR MEDICINE

\title{
Effects of congestive heart failure on plasma von Willebrand factor and soluble P-selectin concentrations in patients with non-valvar atrial fibrillation
}

\author{
G Y H Lip, L A Pearce, B S P Chin, D S G Conway, R G Hart
}

Heart 2005;91:759-763. doi: 10.1136/hrt.2004.036160

See end of article for authors' affiliations

Correspondence to: Professor Gregory Y H Lip, Haemostasis Thrombosis and Vascular Biology Unit, University Department of Medicine, City Hospital, Birmingham B18 7QH; g.y.h.lip@bham.ac.uk

Accepted 28 July 2004
Objective: To examine further the relations of plasma von Willebrand factor ( $v W f$, an index of endothelial damage and dysfunction) and soluble P-selectin (sP-sel, an index of platelet activation) concentrations to the presence and onset of clinical congestive heart failure (CHF) and the degree of left ventricular (LV) dysfunction in patients taking part in the SPAF (stroke prevention in atrial fibrillation) study.

Methods: Plasma concentrations of vWf and sP-sel were measured by enzyme linked immunosorbent assay (ELISA) in 1321 participants in the SPAF III study and related to the presence and onset of clinical $\mathrm{CHF}$, as well as echocardiographic findings. Of the 1321 patients with atrial fibrillation (AF), 331 (25\%) had a documented history of clinical heart failure, of which 168 cases were related to a new or recurrent episode of acute decompensated heart failure occurring within the preceding three months.

Results: Mean plasma vWf was higher among patients with AF and CHF (154 (29) v 144 (31) IU/dl, $\mathrm{p}<0.001$ ), particularly those with acute or recent decompensated symptoms. Patients with severe LV dysfunction on two dimensional echocardiography and low fractional shortening also had significantly higher vWf concentrations than those with no LV dysfunction. CHF patients with clinical features - with (156 (28) IU/dl) and without (152 (31) IU/dl) LV dysfunction-also had higher mean vWf concentrations than patients with asymptomatic LV dysfunction (146 (31) IU/dl, $p<0.001$ ). The presence of mitral regurgitation in $\mathrm{CHF}$ was associated with lower vWf concentrations. Plasma sP-sel concentrations were not affected by presence, onset, or severity of heart failure.

Conclusions: CHF may contribute to hypercoagulability and thrombotic risk in AF through increased endothelial damage and dysfunction. Patients with acute or recent decompensated features have the highest degree of endothelial damage and dysfunction. The presence of CHF clinical features was an important determinant of plasma $\mathrm{vWf}$ concentrations.
$T^{1}$ he risk of stroke and thromboembolism is substantially increased in the presence of non-valvar atrial fibrillation $(\mathrm{AF})$, but if congestive heart failure (CHF) or moderate to severe left ventricular (LV) systolic dysfunction is also present, this risk is further increased two- to fourfold. ${ }^{1}$ In these patients, the source of embolism is presumed to be mural thrombus formation in the left atrium (including the left atrial appendage, especially with concurrent $\mathrm{AF})^{23}$ or left ventricle. ${ }^{4}$ Furthermore, the presence of dilated cardiomyopathy per se also predisposes to left atrial thrombus formation. ${ }^{5}$

Traditionally, abnormal stasis within the atria or ventricle in $\mathrm{AF}$ and $\mathrm{CHF}$, respectively, has been thought to be the main cause of thrombogenesis. However, such abnormal flow is only one component of Virchow's triad of thrombogenesisin recent years, abnormal haemostatic factors and platelets (abnormal blood constituents) and endothelial or endocardial abnormalities (abnormal vessel wall) have been recognised in both AF and CHF, fulfilling Virchow's triad. ${ }^{6-9}$

We recently reported plasma concentrations of von Willebrand factor (vWf, a marker of endothelial damage and dysfunction ${ }^{10}$ ) and soluble P-selectin (sP-sel, a marker of platelet activation ${ }^{11}$ ) in 1321 participants in the SPAF (stroke prevention in atrial fibrillation) III study in relation to stroke risk factors. ${ }^{12}$ In the whole cohort, we found that recent CHF along with age, prior cerebral ischaemia, diabetes, and body mass index were independently associated with vWf and sPsel concentrations. Furthermore, moderate to severe LV dysfunction was also associated with high vWf concentrations on univariate, but not multivariate, analysis of the whole cohort of patients. In the longitudinal analysis, we found that among patients with AF receiving aspirin, raised concentrations of vWf (endothelial damage and dysfunction) were predictive of stroke and vascular events but raised sP-sel concentrations (platelet activation) were not associated with increased cardiovascular risk. ${ }^{13}$ None the less, we were keen to examine further the relations of $\mathrm{vWf}$ and sP-sel concentrations to the presence and onset of clinical CHF and to the degree of LV dysfunction in the CHF subgroup of patients with AF taking part in the SPAF study. Indeed, whether the co-morbid presence of CHF (itself a risk factor for thromboembolism, even when in sinus rhythm) amplifies endothelial damage or dysfunction and platelet activation in patients with AF is uncertain.

\section{PATIENTS AND METHODS}

Baseline venous samples from 1531 patients with non-valvar AF were collected. All patients were participants in SPAF III, which was performed at 20 clinical sites in the USA and Canada between 1993 and 1997; the design and main results have been reported previously. ${ }^{14}$ Patients with any of four high risk criteria (women $>75$ years of age, systolic hypertension $>160 \mathrm{~mm} \mathrm{Hg}$, impaired LV function (clinical heart failure within 100 days of entry or M mode fractional

Abbreviations: $\mathrm{AF}$, atrial fibrillation; $\mathrm{CHF}$, congestive heart failure; ELISA, enzyme linked immunosorbent assay; FS, fractional shortening; $I N R$, international normalised ratio; LV, left ventricular; SPAF, stroke prevention in atrial fibrillation; sP-sel, soluble P-selectin; vWf, von Willebrand factor 
shortening (FS) $\leqslant 25 \%$ ), and previous thromboembolism) were randomly assigned to receive either adjusted dose warfarin (target international normalised ratio (INR) 2-3) or fixed, low dose warfarin (target INR 1.2-1.5) plus aspirin $325 \mathrm{mg} /$ day (termed combination treatment). Participants without any of the four specific risk factors received aspirin $325 \mathrm{mg} /$ day alone. The risk stratification scheme has been clinically validated in several independent assessments. ${ }^{15}$

An earlier study of various haemostatic markers among the same cohort has been reported previously. ${ }^{16}$ Blood samples were initially collected within 30 days of enrolment from all participants and subsequently after three months, 12 months, and annually thereafter. Participants enrolled and followed up at outlying clinics at which specimens could not be adequately processed were not included; thus, one or more samples were collected from 69\% (1339 of 1936) of SPAF III participants for these analyses at baseline or after three months but, owing to natural sample wastage over time, only 1321 of these specimens were available for the present cross sectional analysis. The present study population characteristics are broadly similar to those of the SPAF clinical trial. ${ }^{14}$

\section{Blood collection and laboratory analysis}

Blood collection materials were prepared at the Laboratory for Clinical Biochemistry Research, Department of Pathology, University of Vermont. Blood for vWf and sP-sel assays was drawn into $3.8 \%$ sodium citrate tubes (Becton Dickinson), immediately mixed by gentle inversion, stored on melting ice, and centrifuged at $4^{\circ} \mathrm{C}$ for $30000 \mathrm{~g}$-minutes within one hour of phlebotomy. Plasma was separated for vWf and sP-sel assays. sP-sel and vWf were measured by enzyme linked immunosorbent assay (ELISA) with reagents from R\&D Systems (Abingdon, UK) and Dakopatts (Ely, UK), respectively. The unit for $v W f$ is IU/dl and was standardised by reference vWf from the National Institute for Biological Standards and Controls, Blanche Lane, South Mimms, Potters Bar, Hertfordshire, UK. Intra-assay coefficients of variation for all ELISAs were $<5 \%$ and interassay variances were $<10 \%$.

\section{Assessment of heart failure and LV (dys)function}

Patients entering the SPAF study had heart failure and LV (dys)function defined at each clinical centre clinically and by echocardiography. Firstly, a documented history of (any) clinical CHF was used as a comparison with patients with no documented history of CHF. Of the patients with a history of clinical CHF, patients with a new or recurrent episode of acute decompensated CHF occurring within the preceding three months were compared with patients without such a history. Two dimensional and M mode echocardiography were performed at each study centre. $M$ mode echocardiography was used to calculate FS. The severity of LV dysfunction on two dimensional echocardiography was classed as normal, mild, moderate, and severe and was based on the wall motion index according to the system suggested by the American Society of Echocardiography, as previously described. ${ }^{3}$

\section{Data analysis}

Continuous data were analysed by the Shapiro-Wilks test to determine distribution. Normally distributed data are expressed as mean (SD). Differences in markers between groups were evaluated with two sample $t$ tests and one way analysis of variance, as appropriate, with Tukey's post hoc test for intergroup comparisons. Non-parametric data (sPsel) were determined with normality test and expressed as median (interquartile range). Differences in markers were evaluated with Mann Whitney $U$ and Kruskal-Wallis tests as appropriate. Forward and backward stepwise linear regression analyses were used to identify features independently associated with marker concentrations. Statistical analyses were undertaken with SPSS software (SPSS Inc, Chicago, Illinois, USA). Significance was accepted at the 0.05 level (two sided).

\section{RESULTS}

Of the 1321 patients with AF, 331 (25\%) had a documented history of clinical heart failure, of which 168 cases were related to a new or recurrent episode of acute decompensated heart failure occurring within the preceding three months. Echocardiography found normal systolic function in 144 patients with heart failure, and 137 patients with AF not known to have clinical heart failure had underlying LV dysfunction (termed asymptomatic LV dysfunction). Patients with AF with heart failure did not have a higher frequency of prior cerebral ischaemia (transient ischaemic attack or stroke), although there was a higher proportion of other comorbid illnesses (table 1).

\section{Relation of vWf and sP-sel to presence and onset of clinical heart failure}

Patients with clinical heart failure had higher mean vWf $(\mathrm{p}<0.001)$, but not $\mathrm{sP}$-sel $(\mathrm{p}=0.7)$, concentrations than patients with AF without heart failure (table 1). Among patients with heart failure, those with acute or recent decompensated symptoms (in the previous three months) had higher mean plasma vWf concentrations $(p=0.002)$ than patients with chronic stable disease, possibly corresponding with lower FS $(\mathrm{p}=0.001)$ in the decompensated heart failure group (table 2(a)).

\section{Relation of vWf and sP-sel to LV dysfunction}

When patients were categorised according to severity of LV dysfunction on two dimensional echocardiography (normal, mild, moderate, and severe), the combined group with moderate and severe LV dysfunction had higher mean vWf than did patients with AF with normal function ( $\mathrm{p}=0.002)$ (table 2(b)). Patients with severe LV dysfunction also had significantly lower mean FS and systolic and diastolic blood pressures than did the others (that is, normal, mild, and moderate LV dysfunction).

Patients were also divided into quartiles based on mean FS calculated on $M$ mode echocardiography. FS data were available for 1198 of 1321 patients. Mean vWf in the first two quartiles (higher FS) were lower than in the next two quartiles (lower FS) $(\mathrm{p}<0.001)$ (table $2(\mathrm{c}))$. Mean age, systolic and diastolic pressures, and sP-sel concentrations were comparable across subgroups.

\section{Relation of vWf and sP-sel to clinical heart failure and LV dysfunction}

Lastly, we compared patients classified according to presence of clinical heart failure, presence of LV systolic dysfunction on two dimensional echocardiography, both or neither. Patients with both clinical heart failure and LV dysfunction (HF/dysfunction) had the lowest mean FS and blood pressures. Mean vWf in the HF/dysfunction group was highest compared with patients with AF with neither heart failure nor LV dysfunction (non-HF/normal) and with the asymptomatic LV dysfunction group (table 2(d)). Patients with clinical heart failure but normal systolic function ( $\mathrm{HF} /$ normal) also had significantly higher vWf than did the non$\mathrm{HF} /$ normal group $(\mathrm{p}=0.03)$ despite comparable FS. In contrast, the asymptomatic LV dysfunction group did not differ in plasma vWf from the HF/normal $(p=0.3)$ and non-HF/normal $(p=0.9)$ groups despite a significantly lower FS. 


\begin{tabular}{|c|c|c|c|}
\hline & With clinical HF & Without clinical HF & p Value \\
\hline Number & 331 & 990 & \\
\hline Age (years) & $70(10)$ & $69(9)$ & 0.3 \\
\hline Men & $226(68 \%)$ & 716 (72\%) & 0.2 \\
\hline \multicolumn{4}{|l|}{ Medical history } \\
\hline Prior cerebral ischaemia & $69(21 \%)$ & $170(17 \%)$ & 0.1 \\
\hline Hypertension & $217(66 \%)$ & $529(53 \%)$ & $<0.001$ \\
\hline Diabetes mellitus & $75(23 \%)$ & $121(12 \%)$ & $<0.001$ \\
\hline Peripheral vascular disease & $40(12 \%)$ & $58(6 \%)$ & $<0.001$ \\
\hline Prior Ml & $89(27 \%)$ & $72(7 \%)$ & $<0.001$ \\
\hline Angina & $74(22 \%)$ & $102(10 \%)$ & $<0.001$ \\
\hline CABG & $62(19 \%)$ & $98(10 \%)$ & $<0.001$ \\
\hline \multicolumn{4}{|c|}{ Drug treatment at time of blood sampling } \\
\hline ACE inhibitor & $218(66 \%)$ & $197(20 \%)$ & $<0.001$ \\
\hline Loop diuretic & $233(70 \%)$ & $96(10 \%)$ & $<0.001$ \\
\hline Warfarin & $232(70 \%)$ & $381(39 \%)$ & $<0.001$ \\
\hline Aspirin & $159(48 \%)$ & $636(64 \%)$ & $<0.001$ \\
\hline Smoking history (former + current) & $195(58 \%)$ & $587(59 \%)$ & 0.9 \\
\hline Systolic BP (mm Hg) & $136(20)$ & $137(20)$ & 0.4 \\
\hline Diastolic BP (mm Hg) & $76(11)$ & $79(10)$ & 0.001 \\
\hline \multicolumn{3}{|c|}{ LV dysfunction on two dimensional echocardiography } & $<0.001$ \\
\hline Normal & $144(44 \%)$ & $853(86 \%)$ & \\
\hline Mild & $59(18 \%)$ & $98(10 \%)$ & \\
\hline Moderate & $58(17 \%)$ & $33(3 \%)$ & \\
\hline Severe & $70(21 \%)$ & $6(1 \%)$ & \\
\hline FS (\%) & $28(11)$ & $37(8)$ & $<0.001$ \\
\hline Left atrial diameter $(\mathrm{cm})$ & $5.0(0.8)$ & $4.6(0.7)$ & $<0.001$ \\
\hline Regurgitation & $251(76 \%)$ & $598(61 \%)$ & $<0.001$ \\
\hline$v W f(I U / d l)$ & $154(29)$ & $144(31)$ & $<0.001$ \\
\hline sP-sel (ng/ml) & $32(26-40)$ & $32(25-41)$ & 0.7 \\
\hline \multicolumn{4}{|c|}{$\begin{array}{l}\text { Parametric data are expressed as mean ( } 1 \mathrm{SD}) \text { and compared by two sample } t \text { test. Dichotomous variables are } \\
\text { expressed as absolute number (percentage) and compared by } \chi^{2} \text {. Non-parametric data are expressed as median } \\
\text { (interquartile range) and compared by Mann Whitney } U \text { test. } \\
\text { BP, blood pressure; CABG, coronary artery bypass grafting; FS, fractional shortening determined on } M \text { mode } \\
\text { echocardiography; HF, heart failure; LV, left ventricular; MI, myocardial infarction; sP-sel, soluble P-selectin; vWf, } \\
\text { von Willebrand factor. }\end{array}$} \\
\hline
\end{tabular}

\section{Correlations and multivariate analyses}

Among patients with AF with clinical heart failure, plasma vWf was higher in those with diabetes (table 3(a)). For patients with AF with heart failure, an association was found between plasma vWf and advancing age (Pearson $r=0.14$, $\mathrm{p}=0.01)$ and plasma sP-sel $(r=0.11, \mathrm{p}=0.04)$. In a multivariate analysis examining 19 variables, recent decompensated symptoms $(p=0.007)$, diabetes $(p=0.009)$, sP-sel $(p=0.03)$, and advancing age $(p=0.006)$ were independently associated with increasing plasma vWf in patients with AF with CHF $\left(r^{2}\right.$ adjusted $\left.=0.07\right)$. For the 168 patients with acute or recent heart failure, no further increases in mean vWf were noted with co-morbidities, but mitral regurgitation was associated with lower plasma concentrations (157 (30) $v 169$ (29) IU/dl, p = 0.04).

Median plasma sP-sel concentrations of patients with CHF were higher among current and past smokers and those with peripheral vascular disease and prior myocardial infarction; a trend was found for diabetes. Patients with prior cerebral ischaemia (stroke and transient ischaemic attacks) tended to have lower sP-sel concentrations (table 3(b)).

\section{DISCUSSION}

vWf is one of several endothelium derived haemostatic mediators, with key roles in platelet aggregation and stabilisation of circulating clotting factors. ${ }^{17}$ Large quantities of vWf are stored in the Weibel-Palade bodies of endothelial cells and can be mobilised rapidly after endothelial cell activation ${ }^{18}$ to result in transient increases of plasma $\mathrm{vWf} .{ }^{10}$ In experimental models at least, endothelial dysfunction has been shown to promote thrombosis, vasospasm, and vessel occlusion. ${ }^{19}$ One recent study found increased concentrations of immunoreactive $\mathrm{vWf}$ in the endothelium over the endocardial cells lining the surface of human left atrial appendage in patients with cardiac disease and further found thrombus formation over sites exhibiting impaired endothelial function. ${ }^{20}$

Thus, persistently increased concentrations of vWf and endothelial damage and dysfunction pose a real risk to thrombosis. Indeed, our recent cross sectional study suggests a relation between vWf and risk stratification for stroke and thromboembolism in AF. ${ }^{12}$ Furthermore, our survival analysis of 994 patients with AF found that plasma vWf concentrations were a significant predictor of both stroke and vascular events, with greatest risk at highest concentrations. ${ }^{13}$ Following adjustment for other clinical predictors, the relation between vWf and stroke became non-significant but vWf remained an independent predictor of vascular events. ${ }^{13}$

In this study, we have shown that clinical CHF substantially increases plasma vWf concentrations in patients with $\mathrm{AF}$ and appears to exert more influence over severity of endothelial damage and dysfunction than underlying LV dysfunction. This is further supported by the fact that plasma vWf is highest among patients with acute or recent decompensated CHF, deemed a particularly high risk subgroup for AF related strokes in the SPAF trial patient population, $^{21}$ although more recent analyses have not reported CHF to be a risk factor. ${ }^{22}{ }^{23}$ Of note, none of the other co-morbid diseases in the present study subgroup resulted in further increases in vWf concentrations.

Clinical heart failure is a syndrome characterised by failure of cardiac output to meet the metabolic demands of tissues in the body, which then triggers a host of compensatory mechanisms to support circulation. Neurohormonal stimulation, one of the main compensatory mechanisms, has been known to cause impaired endothelial function. ${ }^{24}{ }^{25}$ Other pathophysiological states induced in CHF that are likely to 


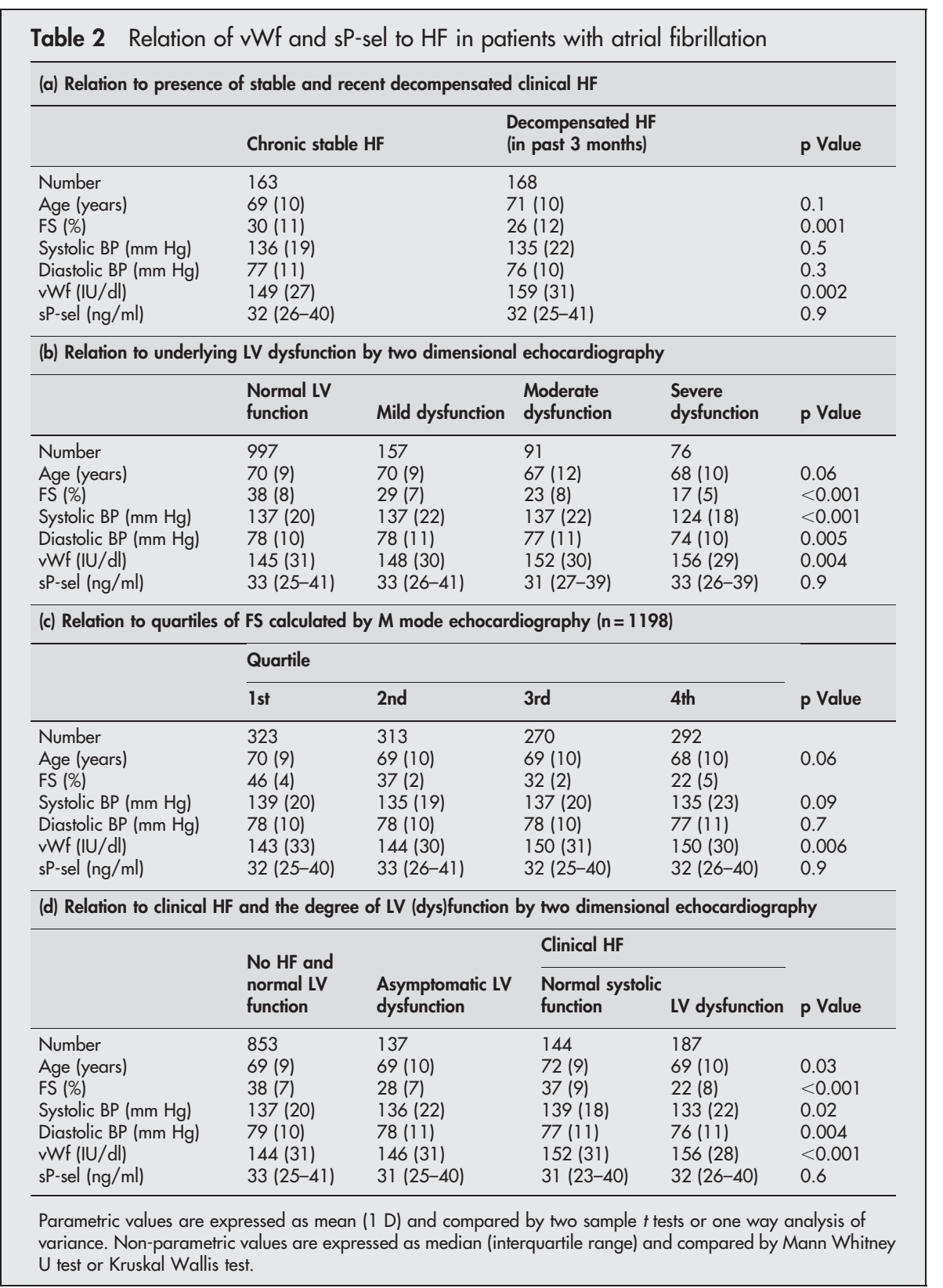

affect endothelial cell function are inflammation and oxidative stress. $^{26}$ In contrast, the presence of mitral regurgitation is associated with lower plasma vWf. This is perhaps not unexpected, as several studies have found a lower incidence of thrombus formation and embolism in patients with heart failure who previously had mitral regurgitation. ${ }^{27-30}$ Indeed, if the decreased thrombus formation in mitral regurgitation may be caused by the stirring effect of the blood pool in the left atrium, then we can hypothesise that a localised endocardial disturbance may prevail, as CHF may well promote more local endothelial damage and dysfunction (for example, by atrial dilatation and distension) rather than as a generalised condition in the entire vasculature.

Unlike vWf, the presence, onset, and severity of heart failure and LV dysfunction had no further effect on plasma sP-sel in patients with AF. One reason may be that platelet activation is more important to thrombogenesis found at sites of high shear stress, such as that occurring in the initial stages of arterial thrombosis, ${ }^{31}$ than to intracardiac thrombus formation where low shear stress predominates. ${ }^{52}$ This would concur with findings in this study of increased sP-sel in patients with peripheral vascular disease and myocardial infarction. Some sP-sel is also released from activated endothelial cells, which may result in the association between sP-sel and vWf concentrations. ${ }^{32}$

This analysis is limited by its cross sectional nature and the lack of a sinus rhythm control group. However, previous studies looking at patients with either AF or heart failure have found plasma vWf and sP-sel to be increased in both groups compared with healthy volunteers in sinus rhythm. ${ }^{83}$ The present analysis is the largest series examining $\mathrm{vWf}$ and sP-sel in patients with AF and concomitant CHF or LV dysfunction. Furthermore, the true pathogenetic mechanisms of thrombogenesis in $\mathrm{AF}$ and $\mathrm{CHF}$ are likely to be more complex than the two aspects studied in the present reportindeed, whether endothelial damage or dysfunction (as expressed by an increased vWF) is local (in the left atrium, for example) or generalised in patients with $\mathrm{AF}$ and $\mathrm{CHF}$ also remains uncertain.

In conclusion, clinical CHF influences plasma concentrations of vWf in AF and may add to the risk of intracardiac 
Table 3 Comparison of plasma markers by associated co-morbidity in 331 patients with atrial fibrillation and HF

\begin{tabular}{|c|c|c|c|}
\hline \multicolumn{4}{|c|}{ (a) vWf (IU/dl) with and without co-morbidity } \\
\hline \multirow[b]{2}{*}{ Co-morbid factors } & \multicolumn{2}{|l|}{ Co-morbidity } & \multirow[b]{2}{*}{ p Value } \\
\hline & Present & Absent & \\
\hline Men & $153(29)$ & $155(31)$ & 0.7 \\
\hline Age $>75$ years & $157(26)$ & $153(31)$ & 0.2 \\
\hline Ever smoked & $154(27)$ & $153(33)$ & 0.7 \\
\hline Prior cerebral ischaemia & $155(27)$ & $154(30)$ & 0.8 \\
\hline Hypertension & $155(29)$ & $152(30)$ & 0.3 \\
\hline Diabetes mellitus & $162(30)$ & $152(29)$ & 0.007 \\
\hline Peripheral vascular disease & $161(25)$ & $153(30)$ & 0.09 \\
\hline Prior Ml & $154(26)$ & $154(31)$ & 0.9 \\
\hline Mitral regurgitation & $153(29)$ & $156(31)$ & 0.6 \\
\hline \multicolumn{4}{|c|}{ (b) sP-sel (ng/ml) with and without co-morbidity } \\
\hline \multirow[b]{2}{*}{ Co-morbid factors } & \multicolumn{2}{|l|}{ Co-morbidity } & \multirow[b]{2}{*}{ p Value } \\
\hline & Present & Absent & \\
\hline Men & $33(26-41)$ & $30(23-39)$ & 0.1 \\
\hline Age $>75$ years & $31(25-39)$ & $33(26-40)$ & 0.2 \\
\hline Ever smoked & $33(26-42)$ & $30(23-39)$ & 0.03 \\
\hline Prior cerebral ischaemia & $31(23-38)$ & $33(26-41)$ & 0.05 \\
\hline Hypertension & $32(25-40)$ & $34(26-41)$ & 0.3 \\
\hline Diabetes mellitus & $34(28-42)$ & $31(25-40)$ & 0.06 \\
\hline Peripheral vascular disease & $37(29-45)$ & $32(25-39)$ & 0.03 \\
\hline Prior Ml & $34(28-44)$ & $32(25-39)$ & 0.03 \\
\hline Mitral regurgitation & $32(25-41)$ & $31(26-39)$ & 0.8 \\
\hline
\end{tabular}

$v W f$ is expressed as mean (1 SD) and compared by two-sample $t$ test. sP-sel is expressed as median (interquartile range) and compared by Mann Whitney $U$ test.

thrombosis and strokes through its effects on endothelial damage and dysfunction. Patients with acute or recent decompensated CHF features have the highest degree of endothelial damage and dysfunction and the presence of clinical heart failure features was an important determinant of $\mathrm{vWf}$ concentrations.

\section{ACKNOWLEDGEMENTS}

The SPAF-III investigators are listed in reference 21. We acknowledge the support of the Dowager Countess Eleanor Peel Trust and the City Hospital Research and Development programme.

\section{Authors' affiliations}

G Y H Lip, B S P Chin, D S G Conway, Haemostasis Thrombosis and Vascular Biology Unit, University Department of Medicine, City Hospital, Birmingham, UK

L A Pearce, Axio Research Corporation, Seattle, Washington, USA R G Hart, University of Texas Health Science Center, San Antonio, Texas, USA

\section{REFERENCES}

1 Atrial Fibrillation Investigators. Risk factors for stroke and efficacy of antithrombotic therapy in atrial fibrillation: analysis of pooled data from five randomised controlled trials. Atrial fibrillation investigators. Arch Intern Med 1994; 154:1449-57.

2 Leung DY, Black IW, Cranney GB, et al. Prognostic implications of left atrial spontaneous echo contrast in nonvalvular atrial fibrillation. J Am Coll Cardiol 1994;24:755-62.

3 SPAF Investigators. Predictors of thromboembolism in atrial fibrillation. II. Echocardiographic features of patients at risk. Stroke prevention in atrial fibrillation investigators. Ann Intern Med 1992;116:6-12.

4 Stratton JR, Resnick AD. Increased embolic risk in patients with left ventricular thrombi. Circulation 1997;75:1004-11.
5 Siostrzonek P, Koppensteiner R, Gossinger H, et al. Hemodynamic and hemorheologic determinants of left atrial spontaneous echo contrast and thrombus formation in patients with idiopathic dilated cardiomyopathy. Am Heart J 1993;125:430-4.

6 Lip GYH. Does atrial fibrillation confer a hypercoagulable state? Lancet 1995;346:1313-4.

7 Lip GYH, Gibbs CR. Does heart failure confer a hypercoagulable state? Virchow's triad revisited. J Am Coll Cardiol 1999;33:1424-6.

8 Gibbs CG, Blann AD, Watson RDS, et al. Abnormalities of hemorheological, endothelial and platelet function in patents with chronic heart failure in sinus rhythm: effects of angiotensin-converting enzyme inhibitor and b-blocker therapy. Circulation 2001;103:1746-51.

9 Jafri SM, Ozawa T, Mammen E, et al. Platelet function, thrombin and fibrinolytic activity in patients with heart failure. Eur Heart J 1993;14:205-12.

10 Lip GYH, Blann AD. von Willebrand factor: a marker of endothelial dysfunction in vascular disorders? Cardiovasc Res 1997;34:255-65.

11 Blann AD, Lip GYH. Is soluble P-selectin a new marker of platelet activation? Atherosclerosis 1997; 128:135-8.

12 Conway DSG, Pearce LA, Chin BSP, et al. Plasma von Willebrand factor and soluble P-selectin as indices of endothelial damage and platelet activation in 1321 patients with non-valvular atrial fibrillation. Circulation 2002; 106:1962-7.

13 Conway DSG, Pearce LA, Chin BSP, et al. Prognostic value of plasma von Willebrand factor and soluble P-selectin as indices of endothelial damage and platelet activation in 994 patients with nonvavular atrial fibrillation. Circulation 2003;107:3141-5.

14 SPAF Investigators. Adjusted-dose warfarin versus low-intensity, fixed-dose warfarin plus aspirin for high-risk patients with atrial fibrillation: stroke prevention in atrial fibrillation III randomised clinical trial. SPAF investigators. Lancet 1996;348:633-8.

15 Hart RG, Halperin JL, Pearce LA, et al. Lessons from the stroke prevention in atrial fibrillation trials. Ann Intern Med 2003;138:831-8.

16 Feinberg WM, Pearce LA, Hart RG, et al. Markers of thrombin and platelet activity in patients with atrial fibrillation: correlation with stroke among 1531 participants in the stroke prevention in atrial fibrillation III study. Stroke 1999:30:2547-53.

17 Roth GJ. Developing relationships: arterial platelet adhesion, glycoprotein Ib, and leucine-rich glycoproteins. Blood 1991;77:5-19

18 Wagner DD. Cell biology of von Willebrand factor. Ann Rev Cell Biol 1990;6:217-46.

19 Luscher TF. The endothelium and cardiovascular disease: a complex relation. N Engl J Med 1994;330:1081-3

20 Fukuchi $M$, Watanabe J, Kumagai K, et al. Increased von Willebrand factor in the endocardium as a local predisposing factor for thrombogenesis in overloaded human atrial appendage. J Am Coll Cardiol 2001;37:1436-42.

21 SPAF Investigators. Predictors of thromboembolism in atrial fibrillation. I. Clinical features of patients at risk. The stroke prevention in atrial fibrillation investigators. Ann Intern Med 1992;116:1-5.

22 Van Walraven C, Hart RG, Wells GA, et al. A clinical prediction rule to identify patients with atrial fibrillation and a low risk for stroke while taking aspirin. Arch Intern Med 2003;163:936-43.

23 Wang TJ, Massaro JM, Levy D, et al. A risk score for predicting stroke or death in individuals with new-onset atrial fibrillation in the community: the Framingham heart study. JAMA 2003;290:1049-56.

24 Francis GS, Benedict C, Johnstone DE, et al. Comparison of neuroendocrine activation in patients with left ventricular dysfunction with and without congestive heart failure. Circulation 1990:82:1724-9.

25 Levine TB, Francis GS, Goldsmith SR, et al. Activity of the sympathetic nervous system and renin-angiotensin system assessed by plasma hormone levels and their relationship to hemodynamic abnormalities in congestive heart failure. Am J Cardiol 1982:49:1659-66.

26 Levine B, Kalman J, Mayer L, et al. Elevated circulating levels of tumor necrosis factor in congestive heart failure. N Engl J Med 1990;323:326-41.

27 Kalaria VG, Passannante MR, Shah T, et al. Effect of mitral regurgitation on left ventricular thrombus formation in dilated cardiomyopathy. Am Heart $J$ 1998;135:215-20

28 Blondheim DS, Jacobs LE, Kotler MN, et al. Dilated cardiomyopathy with mitral regurgitation: decreased survival despite a low frequency of left ventricular thrombus. Am Heart J 1991;122:763-71.

29 Maze SS, Kotler MN, Parry WR. Flow characteristics in the dilated left ventricle with thrombus. J Am Coll Cardiol 1989;13:873-81.

30 Blackshear JL, Pearce LA, Asinger RW, et al. Mitral regurgitation associated with reduced thromboembolic events in high risk patients with non-rheumatic atrial fibrillation. Am J Cardiol 1993;72:840-3.

31 Lowe GDO, Forbes CD. Rheology of cardiovascular disease. In: Lowe GDO, eds. Clinical blood rheology, vol II. Boca Raton: CRC Press, 1988:113-40.

32 Bonfanti R, Furie BC, Furie B, et al. PADGEM (GMP140) is a component of Weibel-Palade bodies of human endothelial cells. Blood 1989;73:1109-12.

33 Li-Saw-Hee FL, Blann AD, Lip GYH. A cross-sectional and diurnal study of thrombogenesis among patients with chronic atrial fibrillation. J Am Coll Cardiol 2000;35:1926-31. 